\title{
The inhibitory effect of tongxieyaofang on rats with post infectious irritable bowel syndrome through regulating colonic par-2 receptor
}

\author{
Xuguang $\mathrm{Hu}^{1^{*}}$, Xiaojun Zhang ${ }^{2}$, Bin $\mathrm{Han}^{1}$ and Weijian Bei ${ }^{1}$
}

\begin{abstract}
Background: The aims of this study were to evaluate the effect and mechanism of a traditional Chinese medicine formula: Tongxieyaofang (TXYF) on Rats with Post Infectious Irritable Bowel Syndrome (PI-IBS).

Methods: SD male rats in adult were used to model PI-IBS and treated with TXYF at three dosage for 14 consecutive days, and then visceral sensation and the frequency of stool in PI-IBS rats were investigated. In addition, the contents of SP, TNF- $a$ and IL-6 in colonic mucosal were analyzed by ELISA. Moreover faecal serine protease activity and PAR-2 mRNA expression were measured by ultraviolet spectrophotometry and RT-PCR, respectively.

Results: Our study showed that TXYF attenuated visceral hyperalgesia and inhibited stool frequency in Campylobacter-stimulated Post Infectious Irritable Bowel Syndrome (PI-IBS) rats. Furthermore, TXYF decreased the colonic SP, TNF- $a$ and IL-6 content in PI-IBS rats. In addition, the up-regulated colonic mucosa PAR-2 mRNA expression in PI-IBS rats was significantly suppressed by orally TXYF.

Conclusions: TXYF attenuated PI-IBS symptom by attenuating behavioral hyperalgesia and anti-diarrhea, the underlying mechanism was mediated by inhibiting PAR-2 receptor expression, reducing the levels of SP, TNF- $a$ and IL-6 in colonic mucosa and decreasing faecal serine protease activity.
\end{abstract}

Keywords: Irritable bowel syndrome, Tong-Xie-Yao-Fang, PAR-2 receptor

\section{Background}

Post-infectious irritable bowel syndrome (PI-IBS) was defined as IBS symptoms occur after an initial episode of acute gastrointestinal infection. Actually, studies demonstrated that $15-32 \%$ of enteric infections led to the generation of new IBS symptoms [1,2], and mounted evidence indicated that previous GI infection or inflammation played an important role in the pathogenesis of IBS [3,4]. Thus, increased attention has been paid to PI -IBS recently, because it was believed that the clear onset and well-defined pathophysiological changes could help us explain the IBS [5].

Tong-Xie-Yao-Fang (TXYF), a prescription in traditional Chinese medicine (TCM), has been widely used

\footnotetext{
* Correspondence: hxquang21@163.com

'School of Traditional Chinese medicine, Guangdong Pharmaceutical University, Guangzhou, 510006, P.R China

Full list of author information is available at the end of the article
}

to relieve symptoms associated with IBS [6]. TXYF composed of atractylodes rhizome, white peony root, dried old orange peel and ledebouriella root (LR) [7]. Our preliminary experimental results demonstrated that TXYF had a significant analgesic effect on IBS rats through regulation the $5-\mathrm{HT}$ in the periphery and CRF in the center [8]. The preliminary clinical study showed that TXYF had the anti-pain and anti-diarrhoea effect on the D-IBS patents [9]. Our recently experimental results also showed that TXYF had the hyperalgesia and anti diarrhoea effect in mice [10]. But the precise mechanism of TXYF has not been fully clear.

Protease activated receptor 2 (PAR-2) is a serine protease receptor which widely distributed in a variety of cells of the digestive tract. Activation of PAR-2 receptor is involved in gut sensation, motility disorders and the intestinal epithelial barrier abnormal. Clinic study showed that there were high level of fecal fluid serine enzyme (a

\section{C) Biomed Central}


protease activation of PAR-2 receptor) and increased contents of SP, IL- 6 and TNF- $\alpha$ in serum of PI-IBS patients. Serine enzyme induced visceral pain sensitivity and intestinal permeability in mouse, and activated the intestinal PAR-2 receptor [11]. Therefore, PAR-2 is suggested to be a new target for drug treatment of IBS [12].

To examine the effect of TXYF and elucidate its mechanism on PI-IBS model, PI-IBS rats were orally treated with TXYF for 14 consecutive days, and then visceral sensation and the frequency of stool were investigated. In addition, the contents of SP, TNF- $\alpha$ and IL-6 in colonic mucosal were analyzed by ELISA. Moreover faecal serine protease activity and PAR-2 mRNA expression were measured by ultraviolet spectrophotometry and RT-PCR, respectively.

\section{Methods \\ Material}

Alosetron was purchased from Sigma-Aldrich (St. Louis, MO, USA); SP, TNF- $\alpha$ and IL-6 ELISA measurement kits were obtained from Jiangcheng Bioengineering Institute (Nanjing, China). Universal RT-PCR measurement kit was obtained from Dingguo Bioengineering Institute (Beijing, China), PAR-2 PCR primers was synthesis by Shanghai Sangon Biological Engineering Technology \& Services Co., Ltd. (Shanghai, China).

\section{Animals}

Sprague-Dawley rats were obtained from the Guangdong Experimental Animal Center. All the rats were individually housed in cages containing bedding material on a 12:12-h light-dark cycle (lights on at 08:00) and provided with food and water ad libitum. The studies were performed in accordance with the proposals of the Committee for Research and Ethical Issues of the International Association and were approved by the Committee on the Use of Human and Animal Subjects in Teaching and Research, Guangdong Pharmaceutical University.

\section{Development of PI-IBS model}

The PI-IBS rat model was developed according to previous report [13]. 50 Sprague-Dawley rats were gavaged with a $1 \mathrm{~mL}$ solution containing C. jejuni $81-176$ at $10^{8}$ $\mathrm{CFU} / \mathrm{mL}$ in Brucella broth. Following gavage, all the rats were housed at five per cage. In the first $3 \mathrm{~d}$ after gavage, stool was collected to verify that intestinal colonization in C. jejuni rats had occurred.

After $90 \%$ of the C. jejuni rats no longer had detectable $\mathrm{C}$. jejuni in the stool, they were considered to be in the post-infectious time period. At this point, they were housed for three additional months. During this time, the stool was also cultured to determine if there was any lingering case of Campylobacter.

\section{Campylobacter gavage}

The C. jejuni 81-176 strain used in the gavage of the rats was obtained from freezer stocks, plated on selective media, and incubated for $36 \mathrm{~h}$ under microaerophilic conditions at $42^{\circ} \mathrm{C}$ to create a bacterial lawn. This lawn was then harvested from these plates and suspended in Brucella broth. The concentration of bacteria was estimated spectropho- tometrically and confirmed via serial dilution and plating on selective media. In the $30 \mathrm{~min}$ prior to Campylobacter gavage, rats were gavaged with a $1 \mathrm{~mL}$ solution of $5 \%$ sodium bicarbonate using a balltipped inoculating needle. This was done to neutralize gastric acid to increase the likelihood of intestinal colonization of the pathogen. Subsequently, a $1 \mathrm{~mL}$ suspension of $\mathrm{C}$. jejuni in Brucella broth was administered by gavage.

\section{Preparation of TXYF}

TXYF extract was supplied by TianJiang pharmacy company, (Lot No. 1203051, Jiangyin, China). The Compositions of TXYF were as following: $6 \mathrm{~g}$ Rhizoma Atractylodis Macrocephalae, 4 g Radix Paeoniae Alba, 3 g Pericarpium Citri Reticulatae and 3 g Radix Sapshnikoviae as shown in Table 1. The raw materials in TXYF all identified by TianJiang pharmacy company. TXYF extract was examined according to the quality control criteria as previous report [14]. $1 \mathrm{~g}$ extract of TXYF was equivalent to $5.3 \mathrm{~g}$ raw materials in TXYF. The contents of indicator constituents in TXYF extract were as following: paeoniflorin, $38.35 \mathrm{mg} / \mathrm{g}$ extract and atractylenolide-1,47.76 mg/g extract. The extract was freshly prepared with distilled water at the desired concentrations just before use. TXYF 4.8, 2.4 g/ kg and $1.2 \mathrm{~g} / \mathrm{kg}$ were defined as high dosage, medium dosage and low dosage, respectively.

\section{Experimental design}

The experiment aimed to test whether TXYF could attenuate visceral hyperalgesia and remedy diarrheal in Campylobacter-induced PI-IBS rats. Rats were divided into 6 group with 10 rats in each group. The Normal rats (Group 1) were treated with distilled water. The PI-IBS rats were randomly divided into 5 groups and treated with distilled water (Group 2), TXYF-H (Group 3),

\section{Table 1 Herbal compositions of TXYF*}

\begin{tabular}{lc}
\hline Herbal name (Latin name) & Quantity (dry, g) \\
\hline Rhizoma Atractylodis Macrocephalae & 6.0 \\
Radix Paeoniae Alba & 4.0 \\
Pericarpium Citri Reticulatae & 3.0 \\
Radix Sapshnikoviae & 3.0 \\
\hline
\end{tabular}

*Four grams of extract from the prescription are obtained in the manufacturing process, and are clinically used as the daily dose. 
TXYF-M (Group 4), TXYF-L (Group 5) and Alosetron (Group 6) respectively. After 2 weeks treatment, rats were used to measure pain threshold pressure and stool frequency, and the stool was collcectd for total faecal serine protease activity measurmen. Moreover, all the rats subsequently were sacrificed to collect colon samples. A $6 \mathrm{~cm}$ long proximal colon (1-2 cm from caecum) was harvested and divided into 2 parts, the proximal was collected for SP, TNF- $\alpha$ and IL- 6 ELISA, the transverse colon mucosa was also collected for PAR-2 mRNA assay.

\section{Examination of abdominal withdrawal reflex and stool frequency}

Examination of the abdominal withdrawal reflex (AWR) was measured using the procedure modified from previous reports [15]. Briefly, rats were lightly anesthetized with diethyl ether, and a balloon $(4 \mathrm{~cm}$ in length, made from the finger of a latex glove) was inserted through the anus into the rectum and descending colon, attached to a Fogarty catheter. The open end of the balloon was secured to the catheter with thread and wrapped with tape ( $1 \mathrm{~cm}$ wide). Prior to use, the balloon was inflated and left overnight so that the latex could stretch and the balloon could become compliant. The balloon was inserted so that the thread was approximately $1 \mathrm{~cm}$ proximal to the anal sphincter, and was held in place by taping the tubing to the tail. The catheter was attached via a connector to a sphygmomanometer pump and a pressure gauge. The rats were then housed in small Lucite cubicle $(20 \times 8 \times 8 \mathrm{~cm})$ on an elevated plexiglas platform and allowed to wake up and adapt for $30 \mathrm{~min}$. Colorectal distention (CRD) was produced by rapidly inflating the balloon to the desired pressure $(20,40,60$, or $80 \mathrm{~mm} \mathrm{Hg}$ ) for a duration of $10 \mathrm{~s}$. Stimuli were applied in an ascending graded manner (spaced by $4 \mathrm{~min}$ ). The AWR, an involuntary motor reflex similar to the visceromotor reflex, was recorded. The AWR score was assigned as follows: $0=$ no behavioral response to distension, 1 = brief head movements followed by abdominal muscle without immobility, $2=$ contraction of lifting of abdomen, 3 = lifting of abdomen, 4 = body arching and lifting of pelvic structure. Measurements of the AWR by visual observation were reproduced by two blinded observers. The pain threshold pressure (PTP) is defined as the stimulus pressure that evokes a visually identifiable contraction of the abdominal wall. Increasing pressure was applied in steps of $5 \mathrm{~mm} \mathrm{Hg}$ lasting 30 s until pain behavior was displayed or until 80 $\mathrm{mmHg}$ was reached to avoid lasting damage to the animals. The stool frequency in 24 hours of all animals was measured after the PI-IBS rat model was treated for two weeks.

\section{Measurement of faecal serine enzymatic activities}

To measure total faecal serine protease activity, supernatants of faecal homogenates $(5 \mathrm{ml})$ were incubated with $1 \mathrm{ml}$ of reaction buffer $(0.15 \mathrm{M} \mathrm{NaCl}$ and $20 \mathrm{mM}$ Tris$\mathrm{HCl}, \mathrm{pH} 8.3$ ) and $1 \mathrm{ml}$ of $0.5 \%$ azocasein at $40^{\circ} \mathrm{C}$. The reaction was stopped after $20 \mathrm{~min}$ with $1 \mathrm{ml}$ of $10 \%$ trichloracetic acid. Following centrifugation, absorption of the clear supernatant was measured at $366 \mathrm{~nm}$.

\section{Measurement of SP, TNF- $a$ and IL- 6 in colonic mucosa}

Rat colon was washed repeatedly with ice-cold saline solution. The mucosa layer was scraped and homogenized. After centrifuge at $10,000 \mathrm{G}$ for $10 \mathrm{~min}$ at $4^{\circ} \mathrm{C}$, the supernatant was collected and stored at $-80^{\circ} \mathrm{C}$ until use. Colonic mucosal levels of SP, TNF- $\alpha$ and IL-6 were determined using ELISA kits according to the manufacturer's protocol.

\section{Reverse transcriptase-polymerase chain reaction (RT-PCR)}

Total RNA was extracted from colonic mucosa and pulmonary tissue using TRIzol reagent. RNA concentration was adjusted to $1 \mathrm{mg} / \mathrm{ml}$ with RNase-free distilled water. Reverse transcrip-tase-polymerase chain reaction (RTPCR) was performed using an Access Quick RT-PCR System, which combined cDNA synthesis and PCR in a single reaction. Sub- sequnetly, $1 \mu \mathrm{l}$ of total RNA was added to the $49 \mu \mathrm{l}$ final reaction mixture solution. For single-step RT-PCR, reverse transcription $\left(48^{\circ} \mathrm{C}\right.$ for 50 min) was followed by initial denaturation at $95^{\circ} \mathrm{C}$ for $2 \mathrm{~min}$. Cycling profiles used were: denaturing at $95^{\circ} \mathrm{C}$ for $30 \mathrm{~s}$; annealing at $55^{\circ} \mathrm{C}$ for $1 \mathrm{~min}$ and extension at $72^{\circ} \mathrm{C}$ for $2 \mathrm{~min}$. A total of 30 cycles was used, followed by a final extension step of $5 \mathrm{~min}$ at $73^{\circ} \mathrm{C}$. The following oligonucleotide primers were designed: PAR-2, 5'-CACC AGTAAGGGAGAAGTCT-3' (sense), 5'-GG GC AG CA CGTCGTGACAGGT-3' (antisense); and $\beta$-actin, '5-CGT GGGCCGCCCTAGGCACCA-3' (sense) and 5'-TTGGCC TTAGGGTTCAGGGGG-3' (antisense).

Suitable sizes of synthesised cDNA for PAR-2 and $\beta$ actin were 385 and $275 \mathrm{bp}$, respectively. PCR products in each cycle were electrophoresed on $2 \%$ agarose gel. PCR products of the predicted size were stained using ethidium bromide and visualized using an ultraviolet transilluminator. Quantification of each band was performed using Scion Image densitometry analysis software.

\section{Statistical analysis}

Results are expressed as mean \pm S.D. The effect of TXYF and LR was examined using one-way ANOVA and Dunnett's test. Differences were considered significant when $\mathrm{P}<0.05$. 


\section{Results}

\section{Effects of TXYF on colon hypersensitivity}

As shown in Figure 1a, the AWR scores in response to graded CRD $(20,40,60$, and $80 \mathrm{mmHg})$ were significantly elevated in PI-IBS rats when compared to that of the normal rats $(\mathrm{P}<0.05)$. TXYF significantly reduced AWR scores in PI-IBS rats.

As shown in Figure 1b, The PTP of PI-IBS rats in response to CRD was reduced when compared to that of the normal rats $(\mathrm{P}<0.05)$. TXYF at three dosage significantly increased The PTP compared to that of PI-IBS rats $(\mathrm{P}<0.05)$. These results suggest that TXYF has analgesic effects on PI-IBS rats in a dosedependent manner.

\section{Effects of TXYF on frequency of stool}

As shown in Figure 2, the frequency of stool was significantly increased in PI-IBS rats when compared to that of the normal rats $(\mathrm{P}<0.05)$, TXYF significantly reduced the frequency of stool in PI-IBS rats $(\mathrm{P}<0.05)$.

\section{Effects of TXYF on faecal serine enzymatic activities}

As shown in Table 2, the serine enzymatic activities were significantly increased in PI-IBS rats when compared to that of the normal rats $(\mathrm{P}<0.05)$, TXYF significantly decreased the enzymatic activities in PI-IBS rats $(\mathrm{P}<0.05)$.

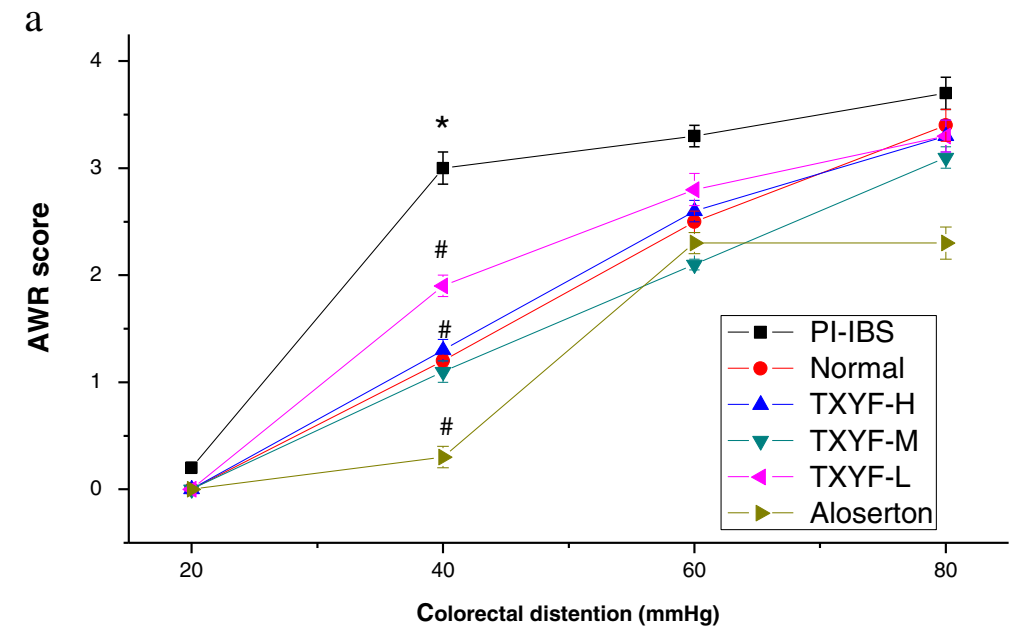

b

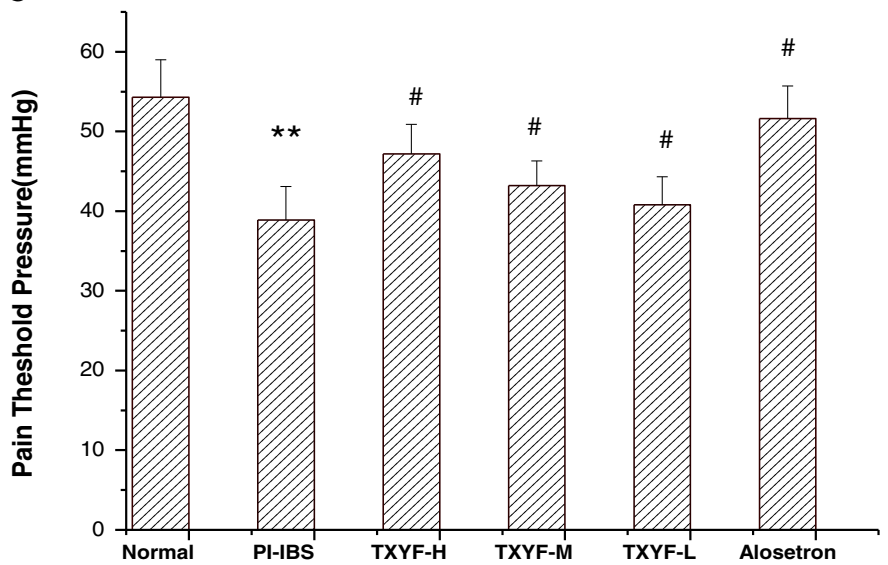

Figure 1 Effects of TXYF on colon hypersensitivity. (a) The effect of TXYF on AWR score in PI-IBS rats. Abdominal withdrawal reflex score (AWR score) measured in response to graded colorectal distension shows significant differences between the PI-IBS group and the normal group and between TXYF treatment group and the PI-IBS group at $40 \mathrm{~mm}$ Hg. Data are mean \pm S.D. ${ }^{* P}<0.05$ vs. Normal, ${ }^{*}$ P $<0.05$ vs. PI-IBS. (b) The effect of TXYF on PTP in PI-IBS rats. Pain threshold pressure (PTP) measured showed significant decrease in the rats with visceral hypersensitivity comparing to the normal control group, and a significant increase in the TXYF at three dosage treatment group comparing to the PI-IBS group. Data are mean \pm S.D. ${ }^{* * P}<0.01$ vs. Normal, ${ }^{\#}$ P $<0.05$ vs. PI-IBS. 


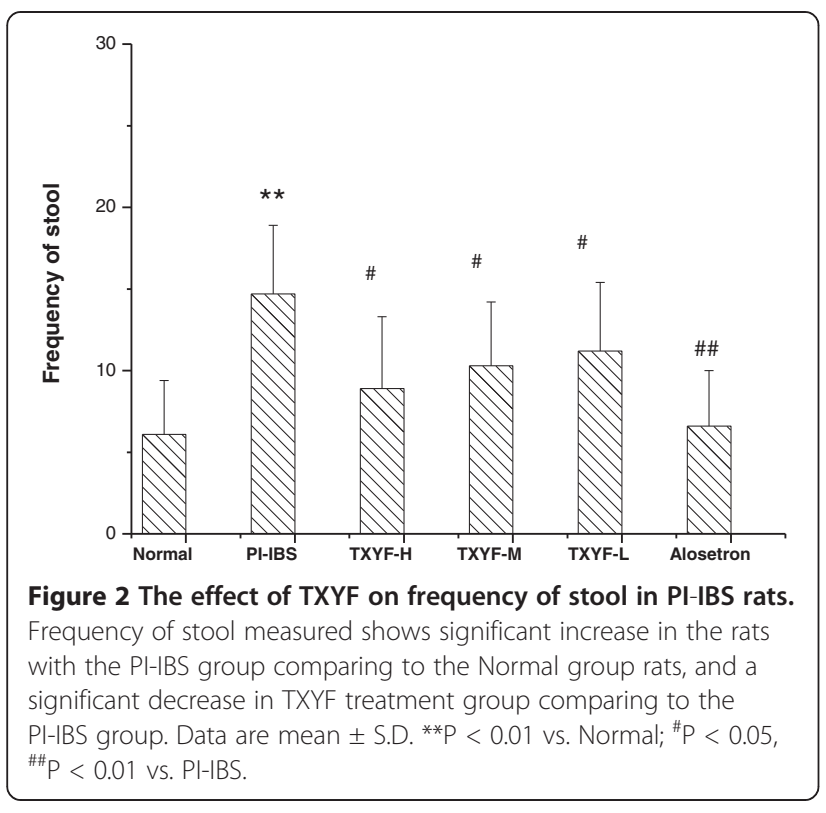

Effects of TXYF on SP, TNF- $a$ and IL- 6 expression in colonic mucosa

As shown in Figure 3, the contents of SP, TNF- $\alpha$ and IL-6 were significantly elevated in PI-IBS rats when compared to that of the normal rats $(\mathrm{P}<0.05)$. TXYF treatment could significantly reduce the colonic mucosa contents of SP, TNF- $\alpha$ and IL- 6 when compared to that of PI-IBS rats $(\mathrm{P}<0.05)$.

\section{Effects of TXYF on PAR-2 expression in the colonic mucosa of PI-IBS rats}

As shown in Table 3 and Figure 4, relative quantification of PAR-2 mRNA in the colonic mucosa revealed that rats undergone PI-IBS had higher levels of PAR-2 mRNA $(1.485 \pm 0.537)$ than that of normal rats $(0.634 \pm$ $0.224)(\mathrm{P}<0.05)$. After TXYF treatment, relative expression quantification of PAR-2 mRNA in the colonic mucosa significantly decreased when compared to that of PI-IBS rats $(P<0.05)$. These results suggest TXYF has analgesic and anti-diarrhea effects on PI-IBS rats in a dose-dependent manner by down-regulating the expression of PAR-2 mRNA in the colonic mucosa.

Table 2 The effect of TXYF on faecal serine enzymatic activities

\begin{tabular}{lccc}
\hline Group & Dose $(\mathbf{g} / \mathbf{k g})$ & No. of rats & Serine activitie $(\mathbf{m g} /$ protein) \\
\hline Normal rats & - & 10 & $772 \pm 236$ \\
PI-IBS rats & - & 10 & $1956 \pm 411^{* *}$ \\
TXYF-H rats & 4.8 & 10 & $1023 \pm 255^{\#}$ \\
TXYF-M rats & 2.4 & 10 & $1254 \pm 289^{\#}$ \\
TXYF-L rats & 1.2 & 10 & $1769 \pm 213$ \\
Alosetron rats & $1.5 \times 10^{-3}$ & 10 & $1235 \pm 256^{\#}$ \\
\hline
\end{tabular}

${ }^{* *} \mathrm{p}<0.01$ vs Normal rats; ${ }^{*} \mathrm{p}<0.05$ vs PI-IBS rats.

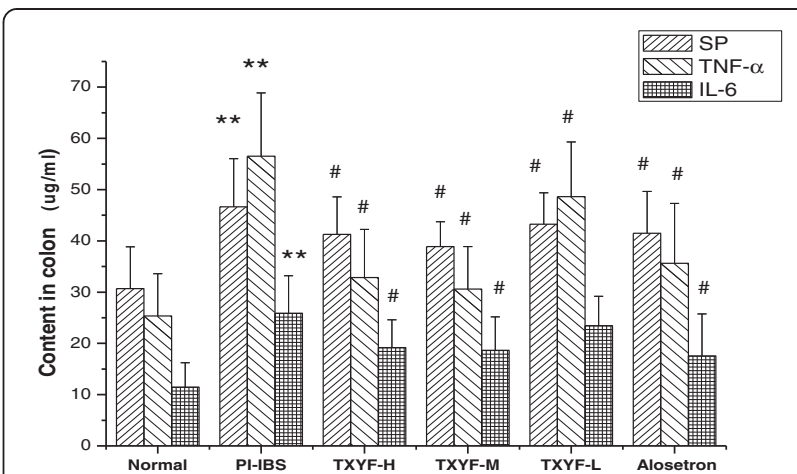

Figure 3 The effect of TXYF on the contents of SP, TNF- $a$ and IL- 6 in colonic mucosa The contents of SP, TNF- $a$ and IL- 6 in colonic mucosa show significant differences between PI-IBS and Normal group and between TXYF treatment group and the PI-IBS group. Data are mean \pm S.D. ${ }^{* *} \mathrm{P}<0.01 \mathrm{vs}$. Normal, ${ }^{\#} \mathrm{P}<0.05$ vs. PI-IBS.

\section{Discussion}

This study revealed that Chinese herbal formula TXYF and its componet herb LR could attenuate visceral hyperalgesia and reduce the frequency of stool in PI-IBS rats, which effect possibly mediated through downregulating the PAR-2 mRNA expression in colonic mucosa.

IBS has a complex etiology and its pathogenesis is related to the altered gut sensory-m otor function, intestinal permeability, and infection, especially low-grade inflammation of the intestine [16]. Epidemiological studies show that bacterial gastroenteritis precedes the onset of the disorder in about $25 \%$ of patients with IBS $[17,18]$. Subtle immune activation has been reported in the colonic biopsies, and altered peripheral cytokine, such as IL- 6 and TNF- $\alpha$ has been shown in PI- IBS patients [19].

Pathophysiological mechanisms of PI-IBS are linked with change in gut flora, altered small intestinal permeability, sensory-motor function and muscle hypercontractility [20]. Recent studies suggest that low-grade inflammation plays important role in development of

Table 3 The effect of TXYF on PAR-2 mRNA expression in the colonic mucosa of PI-IBS rats $(n=10)$

\begin{tabular}{lcc}
\hline Group & $\begin{array}{c}\text { Dose } \\
\mathbf{( g / k g )}\end{array}$ & $\begin{array}{c}\text { Relative expression quantification } \\
\text { of PAR-2mRNA }\end{array}$ \\
\hline Normal rats & - & $0.634 \pm 0.224$ \\
PI-IBS rats & - & $1.485 \pm 0.537^{* *}$ \\
TXYF-H rats & 4.8 & $0.912 \pm 0.197^{\#}$ \\
TXYF-M rats & 2.4 & $1.104 \pm 0.285^{\#}$ \\
TXYF- L rats & 1.2 & $1.206 \pm 0.318$ \\
Alosetron rats & $1.5 \times 10^{-3}$ & $0.903 \pm 0.175^{\#}$ \\
\hline
\end{tabular}

** $p<0.01$ vs Normal rats; ${ }^{*} \mathrm{p}<0.05$ vs PI-IBS rats. 


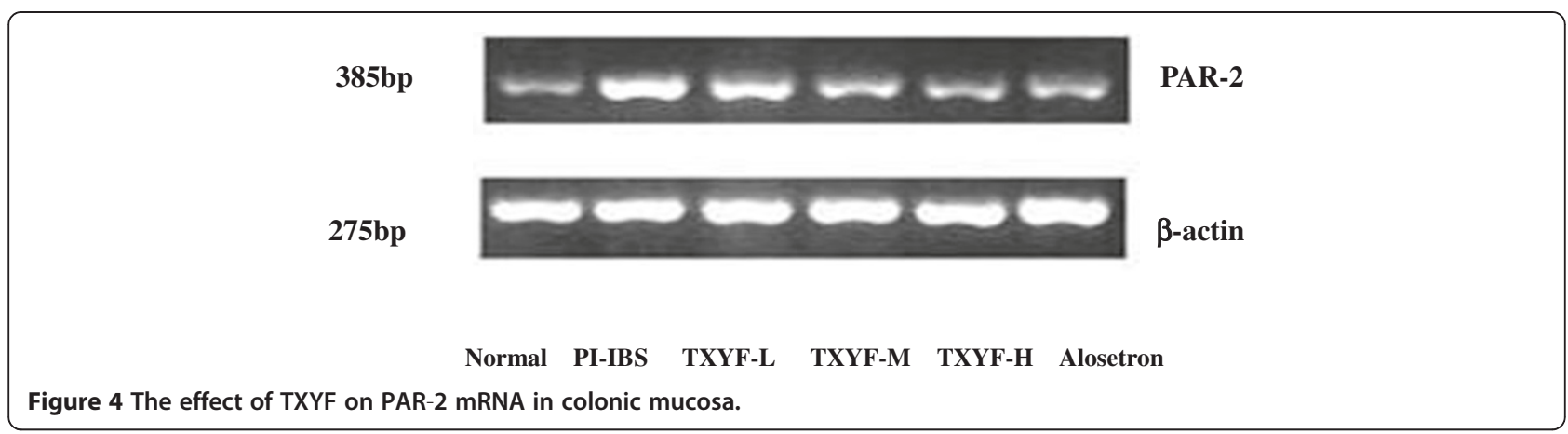

IBS following acute gastroenteritis [21,22], and neuroendocrine factors affect the motor function of gut [23]. Persistently increased number of inflammatory cells in IBS patents has been accompanied with the high expression of TNF- $\alpha$ and IL- 6 mRNA even three months after an acute infective diarrhea [24]. Bood levels of IL-6 and the genetic polymorphism of TNF- $\alpha$ are significantly elevated in IBS patents $[25,26]$. TNF- $\alpha$ and IL-6 are regarded as the most important inflammatory cytokines in IBS. Moreover, the levels of IL-8、IL-10、IL-1 $\beta$ and TGF-1 $\beta$ are significantly increased in IBS patents, especially IL-1 $\beta$ expression is clearly elevated in postinfectious IBS (PI-IBS) [27].

PARs are coupled to G-proteins and cleaved by proteolysis, releasing a tethered ligand domain that binds and activates the receptor. PAR-2 has been reported to be activated by trypsin or mast cell tryptase. PAR-2 is distributed throughout the gastroin testinal tract, where it localized in epithelial cells, myocytes and enteric neurons [28]. Previous clinical data showed that PAR-2 activation is responsible for sensitization of sensory neurons, as well as visceral hypersensitivity in patiets with IBS. These results support the concept that PAR-2 is possibly an important receptor involved in the visceral nociceptive response and intestinal movement disorder. Reduced colonic microflora obtained by oral antibiotic treatment resulted in a lower serine protease activity and was associated with a decreased expression of PAR-2 on the colonic epithelial cells of mice [29]. In the present study, our results showed that the protease activity increased and the PAR-2 mRNA expression was upregulated in PI-IBS rats. Moreover, TXYF could downregulate the PAR-2 mRNA expression and inhibit the serine protease activtate.

Our data suggested that there were the obviosurluy increasment of SP, TNF- $\alpha$ and IL- 6 contents in colonic mucosa of PI-IBS rats. These showed that PAR-2 was activated and then induced the realease of inflammation medium. TXYF could reduce the realease of SP, TNF- $\alpha$ and IL-6. In agreement with our present study, Gecse K found that increased faecals tryptase activity in IBS patent could induce visceral hypersensitivity in mice [28].
The mechanism for this phenomenon is that trypsin activates PAR-2 receptor increasing the excitability of dorsal root ganglion neurons and promoting SP and CGRP release. Tanaka $Y$ found the infection factors of gastrointestinal can activate PAR-2 receptor promoting inflammation medium release, such as IL-6, IL- 8 and TNF- $\alpha[30]$.

Previous research showed that TXYF can inhibit neonatal colon irritation-induced IBS by decreasing 5 -HT in the serum and decreasing SP and CGRP in the plasma [31]. TXYF can treat IBS probably by affecting the secretion and release of gastrointestinal hormone (VIP) after the partial restriction stress binding stimulation in the IBS rat model [32]. TXYF has a marked inhibitory effect on the degranulation of peritonea1 mast cells in sensitized rats induced with $\mathrm{C} 48 / 80$, which indicates that TXYF exerts therapeutic effect by inhibiting enteric mast cell activation and thus decreasing histamine release, which in part explains the mechanism of TXYF in IBS [33].

In conclusion, our present research found that TXYF played an important role in attenuating behavioral hyperalgesia and anti-diarrheal effect. Down-regulating PAR-2 expression and reducing the level of cytokines in the colonic mucosa could be the possible molecular mechanism.

\section{Conclusions}

Our study demonstrated that TXYF attenuated PI-IBS symptom by attenuating behavioral hyperalgesia and anti-diarrhea, the underlying mechanism was mediated by inhibiting PAR-2 receptor expression, reducing the levels of SP, TNF- $\alpha$ and IL- 6 in colonic mucosa and decreasing faecal serine protease activity.

\section{Competing interests}

The author's declare that they have no competing interests.

\section{Authors' contributions}

$\mathrm{XH}$ designed the study and coordinated the experiments and draft of the manuscript. BH carried out the animal study. XZ participated in the experimental design and data analyses. WB conducted the extraction of RNA and RT-PCR. XH measured the contents of SP, TNF- $a$ and IL-6 in colonic mucosal by ELISA. BH participated in the design and analysis of the 
experiment. $X Z$ involved in discussion of experiment and draft of the manuscript. All authors read and approved the final manuscript.

\section{Acknowledgements}

The present study was supported by a Grant from the National Science Council (NSC 81173194) of China.

\section{Author details}

${ }^{1}$ School of Traditional Chinese medicine, Guangdong Pharmaceutical University, Guangzhou, 510006, P.R China. ${ }^{2}$ School of Pharmacy, Guangzhou University of Chinese Medicine, Guangzhou, P.R China.

Received: 4 July 2013 Accepted: 26 September 2013 Published: 2 October 2013

\section{References}

1. Dunlop SP, Jenkins D, Neal KR, Spiller RC: Relative importance of enterochromaffin cell hyperplasia, anxiety, and depression in post infectious IBS. Gastroenterology 2003, 125:1651-1659.

2. Gwee KA, Leong YL, Graham C: The role of psychological and biological factors in post infective gut dysfunction. Gut 1999, 44:400-406.

3. Spiller R, Garse K: Post infectious irritable bowel syndrome. Gastroen terology 2009, 136:1979-1988

4. Kok-Ann G, FRCP Ph D: Post-infectious irritable bowel syndrome, an inflammation-immunological model with relevance for other IBS and functional dyspepsia. J Neurogastroenterol Motil 2010, 16:130-134.

5. Spiller R, Garsed K: Infection, inflammation, and the irritable bowe syndrome. J Digestiver Disease 2009, 41:844-849.

6. Fan H, Qiu MY, Mei JJ, Shen GX, Liu SL, Chen R: The study of Tong-XieYao-Fang on 52 cases of D-IBS patients. World J Gastroenterol 2005 11:4800-4806

7. Liao H, Banbury LK, Leach DN: Elucidation of DanzhixiaoyaoWan and its constituent herbs on antioxidant activity and inhibition of nitric oxide production. eCAM 2007, 4:425-430.

8. Xu-guang $H$, Di X, Yue Z, Xiang-bo Y, Han S: The alleviating pain effect of aqueous extract from Tong-Xie-Yao-Fang, on experimental visceral hypersensitivity and it mechanism. Biol Pharm Bull 2009, 32:1079.

9. Xu-guang $\mathrm{H}$, Zhao-Hui W: The clinic study of Tong-Xie-Yao-Fang on stagnation and spleen deficiency in 35 D-IBS patients. Xinzhongyi 2012, 23:1075-1079.

10. Xu-guang Hu B-H: The effect of Ledebouriella root in Tong-Xie-Yao-Fang. Chinese J Modern Drug Application 2012, 8:46-48.

11. Cenac N, Andrews CN, Holzhausen M, Chapman KG, Beck P, Bunnett NW: Role for protease activity in visceral pain in irritable bowel syndrome. Clin Invest 2009, 117:636-647.

12. Bueno L: Protease activated receptor 2: a new target for IBS treatment. Eur Rev Med Pharmacol Sci 2008, 15:95-102.

13. Sam Ryong J, Walter M, Kimberly L: ICC density predicts bacterial overgrowth in a rat model of post-infectious IBS. World J Gastroenterol 2010, 16:3680-3686.

14. Song R, Ping W: The study on quality standard of TongXieYaoFang Granules. Pharm J Chin PLA 2012, 28:154-156.

15. Al-Chaer ED, Kawasaki M, Pasricha PJ: A new model of chronic visceral hypersensitivity in adult rats induced by colon irritation during postnatal development. Gastroenterology 2000, 119:1276-1285

16. Ghoshal UC, Park H, Gwee KA: Bugs and irritable bowel syndrome: the good, the bad and the ugly. J Gastroenterol Hepatol 2010, 25:244-251.

17. Ghoshal U, Ranjan P: Post-infectious irritable bowel syndrome: the past, the present and the future. J Gastroenterology 2011, 8:94-101.

18. Dunlop SP, Jenkins D, Spiller RC: Distinctive clinical, psychological,and histological features of postinfective irritable bowel syndrome. Am J Gastroenterol 2003, 98:1578-1583

19. O'Mahony L, Walter J, Komjerly L: Lactobacillus and bifidobacterium in irritable bowel syndrome: symptom responses and relationship to cytokine profiles. Gastroenterology 2005, 128:541-551.

20. Cherbut C, Aube AC, Blottiere HM, Galmiche JP: Effects of short-chain fatty acids on gastrointestinal motility. Scand J Gastroenterol 1997, 222:558-561.

21. Nystedt S, Emilsson K, Wahlestedt C, Sundelin J: Molecular cloning of a potential proteinase activated receptor. Proc Natl Acad Sci 2009, 91:9208-9212.
22. Long $Y Q$, Tong JJ, Qian W, Hou XH: Altered expression of intestinal cytokines in development of post infectious irritable bowel syndrome mouse model. Zhonghua Yi Xue Za Zhi 2010, 90:2415-2419.

23. Akiho H, Deng $Y$, Blennerhassett $P$, Kanbayashi H, Collins SM: Mechanisms underlying the maintenance of muscle hypercontractility in a model of postinfective gut dysfunction. Gastroenterology 2005, 129:131-141.

24. Gecse K, Róka R, Ferrier L: Increased faecal serine protease activity in diarrhoeic IBS patients: a colonic lumenal factor impairing colonic permeability and sensitivity. Gut 2008, 57:1035-1037.

25. Rana SV, Sharma S, Sinha SK, Parsad KK, Malik A, Singh K: Pro-inflammatory and anti-inflammatory cytokine response in diarrhoea-predominant irritable bowel syndrome patients. Trop Gastroenterol 2012, 33:251-256

26. Swan C, Duroudier NP, Campbell E, Zaitoun A, Hastings M, Dukes GE, Cox J, Kelly FM, Wilde J, Lennon MG, Neal KR, Whorwell PJ, Hall IP, Spiller RC: Identifying and testing candidate genetic polymorphisms in the irritable bowel syndrome (IBS): association with TNFSF15 and TNF-a. Gut 2013, 62:985-994.

27. Scalera A, Minno MNDD, Tarantino G: What does irritable bowel syndrome share with non-alcoholic fatty liver disease? World J Gastroenterol 2013, 33:5402-5420.

28. Reed DE, Barajas-Lopez C, Cottrell G, Velazquez-Rocha S, Dery O, Grady EF, Bunnett NW, Vanner SJ: Mast cell tryptase and proteinase-activated receptor 2 induce hyperexcitability of guinea-pig submucosal neurons. J Physiol 2003, 547:531-542.

29. Cenac N, Coelho AM, Nguyen C, Compton S, Ndrade-Gordon P, MacNaughton WK, Wallace Bueno L: Induction of intestinal inflammation in mouse by activation of proteinase-activated receptor-2. Am J Clin Pathol 2007, 161:1903-1915.

30. Tanaka Y, Sekiguchi F, Hong H, Kawabata A: PAR-2 triggers IL-8 release via MEK/ERK and $\mathrm{Pl}_{3}$-kinase/Akt pathway in $\mathrm{Gl}$ epithelial cells. Biochem Biophys Res Commun 2008, 377:612-622.

31. Zhao YB, Li DG: The effect of Tong-Xie-Yao-Fang on neonatal colon irritation induced visceral hypersensitivity. Chinese Traditional Patent Medicine 2010, 28:852-855

32. Li DH, Li CM, Li WS, Zhou ZD, Zhang Y: The effect of TXYF on restriction stress binding induced IBS rat model. Lishizhen Medicine Maternal Med Res 2009, 12:2036-2040.

33. Liu ZG, Zhao YB: The inhibitory effect of TXYF on the degranulation of mast cells in IBS rats. Modern J Integrated Trad Chin Western Med 2011, 18:1723-1726.

\section{doi:10.1186/1472-6882-13-246}

Cite this article as: Hu et al:: The inhibitory effect of tongxieyaofang on rats with post infectious irritable bowel syndrome through regulating colonic par-2 receptor. BMC Complementary and Alternative Medicine 2013 13:246.

\section{Submit your next manuscript to BioMed Central and take full advantage of:}

- Convenient online submission

- Thorough peer review

- No space constraints or color figure charges

- Immediate publication on acceptance

- Inclusion in PubMed, CAS, Scopus and Google Scholar

- Research which is freely available for redistribution 